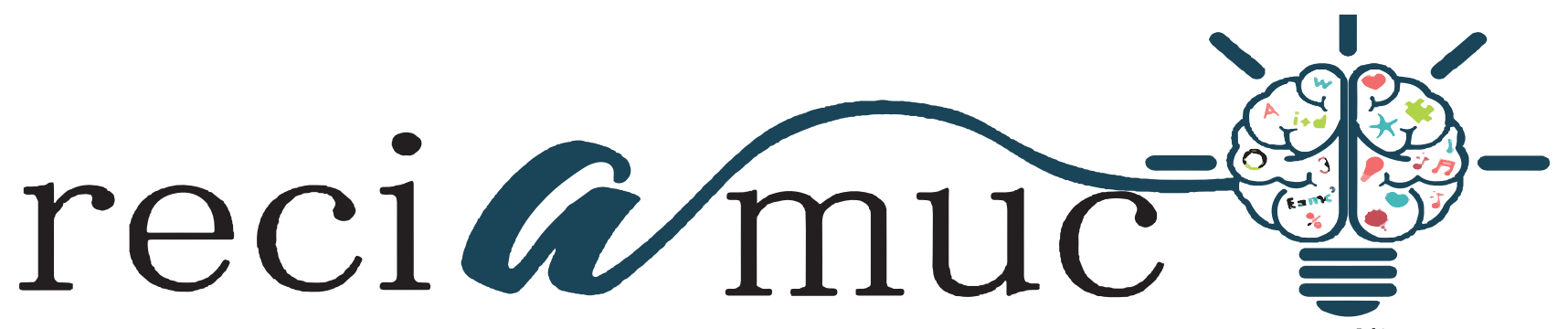

DOI: 10.26820/reciamuc/4.(1).enero.2020.229-241

URL: https://reciamuc.com/index.php/RECIAMUC/article/view/424

EDITORIAL: Saberes del Conocimiento

REVISTA: RECIAMUC

ISSN: $2588-0748$

TIPO DE INVESTIGACIÓN: Artículo de Revisión

CÓDIGO UNESCO: 5802 Organización y Planificación de la Educación

PAGINAS: $229-241$

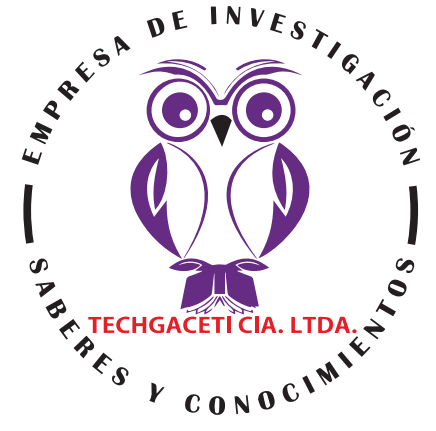

\title{
El alumnado de la Universidad de Guayaquil (UG). Un vistazo al equilibrio de los sexos
}

The students of the University of Guayaquil (UG).
A look at the balance of the sexes

Os estudantes da Universidade de Guayaquil (UG). Um olhar sobre o equilíbrio dos sexos

Eva María de Lourdes Loaiza Massuh'; Patricia Maria Salazar Torres²; Richard Romero Izurieta ${ }^{3}$

RECIBIDO: 18/11/2019 ACEPTADO: 20/12/2019 PUBLICADO: 31/01/2020

1. Magister en Educación Superior; Licenciada en Ciencias de la Educación; Universidad de Guayaquil; Guayaquil, Ecuador; eva.loaizam@ug.edu.ec; (iD https://orcid.org/0000-0002-4041-8597

2. Magister en Contaduría Pública; Magister en Dirección Administrativa y Financiera; Contadora Públical; Universidad de Guayaquil; Guayaquil, Ecuador; patricia.salzarto@ug.edu.ec; (DD https://orcid.org/0000-0003-41833765

3. Magister en Administración de Empresas mención en Negocios Internacionales; Ingeniero en Computación; Universidad de Guayaquil; Guayaquil, Ecuador; richard.romeroi@ug.edu.ec; (D) https://orcid.org/0000-00023387-6661

CORRESPONDENCIA

Eva María de Lourdes Loaiza Massuh

eva.loaizam@ug.edu.ec

Guayaquil, Ecuador 


\section{RESUMEN}

Es necesario reconocer que nuestra historia común ha dejado inexorables evidencias de la desigualdad que el género femenino ha tenido que soportar y sobrellevar en gran parte del desarrollo de nuestra civilización, de allí pues, es entonces comprensible la inconmensurable e indetenible demanda que dicha representación le ha tocado mantener, cada vez con mayor énfasis y frecuencia, en los diversos ámbitos de la vida contemporánea a nivel mundial. El presente trabajo investigativo, realizado en base a un diseño bibliográfico y mediante una metodología de revisión, se enfoca en exponer sobre lo que la literatura encontrada refiere respecto al equilibrio de los sexos a nivel universitario, en un contexto amplio y luego en lo que respecta a la Universidad de Guayaquil (UG). Con los resultados obtenidos se ha logrado exponer, entre otros aspectos, sobre las acepciones que algunos autores, desde una perspectiva general y de la educación superior, sostienen sobre la temática implícita en cuanto al equilibrio de los sexos, equidad y/o igualdad de géneros, tendencia mundial, contexto ecuatoriano y, finalmente, lo que se percibe en términos tangibles como actuaciones por parte de la UG y sus estudiantes, respecto a esta problemática. Las conclusiones radicaron fundamentalmente en reconocer dos aspectos indiscutibles, pudiendo ser el primero de ellos, el hecho de que, efectivamente, tanto las políticas y estrategias gubernamentales como las de la UG, son percibidas como positivas y bien encaminadas hacia una mejoría en cuanto al cambio del enfoque del sistema educativo superior en favor del equilibrio de género, la inclusión y consideración de las mujeres en diversas esferas socioculturales, políticas y económicas, primordialmente, sin embargo, el otro aspecto que también ha de reconocerse es que, aún y con la tendencia mundial, ni siquiera es soló Ecuador sino toda Latinoamérica está muy lejos de materializar la abanderada transversalidad de género puesto que, la cuestión es más que todo de arraigo cultural en favor de una mentalidad machista por demás dura de vencer, que es reacia a aceptar que la mujer es tan capaz y, muchas más veces de las que se reconoce, productiva que el hombre.

Palabras clave: Civilización; Educación superior; Estrategias gubernamentales; Sistema educativo superior; Transversalidad de género.

\section{ABSTRACT}

It is necessary to recognize that our common history has left inexorable evidence of the inequality that the female gender has had to endure and cope with in large part of the development of our civilization, hence the immeasurable and unstoppable demand that such representation has made it understandable. Touched to maintain, with increasing emphasis and frequency, in the various areas of contemporary life worldwide. This research work, based on a bibliographic design and through a review methodology, focuses on exposing what the literature found refers to the balance of the sexes at the university level, in a broad context and then in what regards to the University of Guayaquil (UG). With the results obtained it has been possible to expose, among other aspects, about the meanings that some authors, from a general perspective and of higher education, hold on the implicit theme regarding gender balance, equity and / or gender equality, global trend, Ecuadorian context and, finally, what is perceived in tangible terms as actions by the UG and its students, regarding this problem. The conclusions were fundamentally in recognizing two indisputable aspects, being the first one, the fact that, in fact, both government policies and strategies and those of the UG, are perceived as positive and well directed towards an improvement in terms of change of the approach of the higher education system in favor of gender balance, the inclusion and consideration of women in various socio-cultural, political and economic spheres, primarily, however, the other aspect that must also be recognized is that, even with the trend In the world, Ecuador is not even alone, but all of Latin America is far from materializing the standardized gender mainstreaming since, the issue is mostly culturally rooted in favor of a macho mentality that is hard to defeat, which is reluctant to accept that the woman is so capable and, many more times than she recognizes herself, productive than the man.

Keywords: Civilization; Higher education; Government strategies; Higher education system; Gender mainstreaming.

\section{RESUMO}

É necessário reconhecer que nossa história comum deixou evidências inexoráveis da desigualdade que o gênero feminino teve de suportar e lidar com grande parte do desenvolvimento de nossa civilização, daí a demanda imensurável e imparável de que essa representação a tornou compreensível . Tocado para manter, com crescente ênfase e frequência, nas diversas áreas da vida contemporânea em todo o mundo. Este trabalho de pesquisa, baseado em um desenho bibliográfico e através de uma metodologia de revisão, tem como foco expor o que a literatura encontrada se refere ao equilíbrio dos sexos no nível universitário, em um contexto amplo e depois no que diz respeito à Universidade de Guayaquil (UG ) Com os resultados obtidos, foi possível expor, entre outros aspectos, os significados que alguns autores, de uma perspectiva geral e do ensino superior, mantêm sobre o tema implícito sobre equilíbrio de gênero, equidade e / ou igualdade de gênero, tendência global, Contexto equatoriano e, finalmente, o que é percebido em termos tangíveis como ações da UG e de seus alunos em relação a esse problema. As conclusões foram fundamentais para reconhecer dois aspectos indiscutíveis, sendo o primeiro, o fato de que, de fato, as políticas e estratégias governamentais e as da UG são percebidas como positivas e bem direcionadas para uma melhoria em termos de mudança de abordagem. do sistema de ensino superior em favor do equilíbrio de gênero, da inclusão e consideração das mulheres em várias esferas socioculturais, políticas e econômicas, principalmente, no entanto, o outro aspecto que também deve ser reconhecido é que, mesmo com a tendência mundial , O Equador nem está sozinho, mas toda a América Latina está longe de materializar a integração padronizada de gênero, uma vez que a questão está principalmente culturalmente enraizada em favor de uma mentalidade machista difícil de derrotar, que reluta em aceitar que a mulher é tão capaz e, muito mais vezes do que ela se reconhece, produtiva que o homem.

Palavras-chave: Civilização; Ensino superior; Estratégias de governo; Sistema de ensino superior; Integração da perspectiva de gênero. 


\section{Introducción}

Exponer sobre el equilibrio de los sexos, en definitiva, incluye tener que abordar una temática variada, pero a la vez interrelacionada. Considerando que, al respecto, también pudiera hacerse referencia a la igualdad de los sexos o de géneros, ha de comprenderse que tales alusiones en síntesis trascienden más allá del hecho de querer alcanzar una simple paridad entre la cantidad de personas de ambos sexos que interactúan en los diversos contextos de la vida social, pues es menester que esto también deba entenderse como una temática que alcanza dimensiones vinculadas a la historia, moral, religión, política, cultura, economía, educación, derecho, razas, valores y un sin número de aspectos, pero fundamentalmente, y no por mencionarla de última es menos importante, es una cuestión de humanidad.

Habría que igualmente reconocer que nuestra historia común ha dejado inexorables evidencias de la desigualdad que el género femenino ha tenido que soportar en gran parte del desarrollo de nuestra civilización, de allí que entonces, es comprensible la inconmensurable e indetenible demanda que dicha representación le ha tocado adelantar, cada vez con mayor énfasis y frecuencia en los diversos ámbitos de la vida contemporánea a nivel mundial, sin menos cabo del apoyo, reconocimiento y reivindicaciones protagonizadas gradualmente por los representantes del sexo opuesto.

Por su parte, Baute, Pérez, \& Espinoza (2017) con su obra dejan claro que, para poder estimar la materialización la igualdad de género y el empoderamiento de la mujer, resulta indiscutible la concreción de un orden internacional justo y equitativo, que se traduzca en el fin de las guerras, en la priorización al ser humano ante el capital, en la supresión la pobreza y el hambre, y en la conservación del medio ambiente, eventos que por ende se encuentra estrechamente vinculados al derecho propio de los seres humanos de gozar de una vida digna y un mundo en el que las mujeres sean igualmente protagonistas de su desarrollo, y no solamente beneficiarias de este.

Maqueira \& Recalde (2017) indicaron que han sido trascendentales los cambios que en las últimas décadas se han ido produciendo en el ámbito de la Educación Superior, tanto a nivel mundial como regional, por una parte, debido a la oferta de oportunidades, y por la otra, también a lo que representan los cambios en materia curricular. Agregan que, es en base a nuevas realidades políticas, económicas y sociales originadas del mundo globalizado en el que nos desenvolvemos, que aún existe el reto de "concebir, organizar, coordinar y financiar la formación y educación de los futuros profesionales enfocada en métodos contemporáneos que permitan responder al desarrollo de competencias profesionales con calidad".

Es por ello, que las tendencias contemporáneas en cuanto a Educación Superior (Ruiz, Martínez, \& Valladares, 2012; Atria, 2012; Pupo Ávila, N. L., Pérez, Alfonso, Pérez, \& González, 2013), encuentran puntos comunes en propiciar sistemas más equitativos que respondan a las necesidades del desarrollo económico y social. Se requiere de programas y ofertas que tengan un carácter diferenciado, flexible, adaptable que faciliten la integración de todos los usuarios y beneficiarios en la construcción de aprendizajes significativos que contribuyan a transformar la realidad social. A partir de la Ley Orgánica de Educación Superior, (LOES), se emiten los principios que rigen de manera integral las instituciones, actores, procesos, normas, recursos, y demás componentes del sistema de Universidades, los cuales derivan en Modelos Educativos que sustentan la igualdad de oportunidades, la calidad, pertinencia, integralidad, la producción científica entre otros aspectos (Foster \& Norton, 2012; Díaz, Fernández-Cid, Gómez, Toboso, Vázquez, \& Villa, 2012; Bol \& Van de Werfhorst, 2013). (Maqueira \& Recalde, 2017). 
El presente trabajo investigativo se enfoca en exponer sobre lo que la literatura consultada refiere respecto al equilibrio de los sexos a nivel universitario en un contexto amplio y luego en lo que respecta a la UG, procurando además rescatar aquellas fuentes bibliográficas que de alguna manera también puedan servir de referencia para exponer qué sucede en el entorno del alumnado de la UG respecto a esta temática. Es por ello que a continuación, se expondrá sobre las acepciones que algunos autores, desde una perspectiva general, y otros, desde una perspectiva de la educación superior, sostienen sobre la temática implícita en cuanto al equilibrio de los sexos, equidad y/o igualdad de géneros, su contexto histórico, desigualdad de género, tendencia mundial de la feminización de la educación superior, contexto ecuatoriano sobre la desigualdad en el sistema educativo superior $y$, finalmente, lo que se percibe en términos tangibles como actuaciones por parte de la UG y sus estudiantes, respecto a esta problemática en la realidad reciente.

\section{Materiales y Métodos}

A principios de noviembre de 2019, se llevó a cabo una búsqueda estructurada de literatura cientificoacadémica en bases de datos tales como: Dialnet, EBSCO, ScienceDirect, Embase, SciELO, ResearchGate y otros, con las expresiones "equilibrio de los sexos en la universidad", "igualdad de géneros en la Universidad de Guayaquil" e "igualdad de géneros en la educación superior ecuatoriana" a (2018) fines de identificar aspectos que fundamentaran la situación de la equidad de géneros en el alumnado de la UG.

Los resultados obtenidos de la referida pesquisa fueron limitados, debido a que se evidenció la falta de contenido específico que en base a sus títulos identificativos se relacionaran de manera más directa con la temática planteada.

En base a dicho objetivo, esos resultados se fueron filtrando, escogiendo solo aquellos trabajos investigativos de mayor correlación y/o relevancia respecto a la temática planteada, con acceso completo y gratuito, en idioma español, referentes a literatura tipo: revisiones sistemáticas con o sin meta-análisis, artículos de revisión narrativa, tesis de grado, postgrado y doctorado, ensayos y demás trabajos monográficos con características científico-académicas, desarrolladas en el marco de educación superior y ciencias sociales, fundamentalmente, que hubiesen sido publicados en los últimos 10 años, incluyendo el corriente, es decir, de 2009 a 2019, no obstante, en algunos casos fue necesario hacer excepciones debido a que el equipo investigador consideró que el material aún podría asumirse como vigente y/o con evidencia de destacado valor científico. Se desestimaron editoriales, cartas al editor y otro tipo de materiales bibliográficos que básicamente no cumplían con el carácter científico-académico requerido.

De forma secundaria, se realizó una búsqueda sin limitación cronológica ni del nivel de evidencia para identificar información relevante y necesaria para el desarrollo de la presente revisión. La inclusión o exclusión de cada una de las referencias en la presente revisión se definió por el consenso de los autores de esta obra, al igual que las decisiones discordantes.

\section{Resultados}

\section{Generalidades}

Baute, Pérez, \& Espinoza (2017) aclaran que los temas de género y las inquietudes por las cuestiones en cuanto a igualdad y equidad no son solamente propias de las mujeres, sino también involucran a los hombres, sin exclusiones en cuando a las diferencias de edades, raza, color de piel, preferencia sexual e identidades de género puesto que alcanzan una perspectiva más general. De la misma manera consideran que un componente indispensable para el alcance de la liberación del género femenino y para la concepción cambios en la vida pública y privada precisamente vendría 
siendo la educación.

La mujer vista desde la perspectiva de género y desde adentro del sistema educativo supone, en primer lugar, el reconocimiento de la diferenciación que surge dentro de la interacción de los géneros en las posiciones de poder a las que pertenecen. Son múltiples las organizaciones y autores que abordan el tema de la equidad de género a nivel internacional en la adopción de normas que orienten reformas al Estado en función de ayudar a empoderar a las mujeres y a reclamar sus derechos. El género como herramienta para el análisis social ha sido incorporado de manera masiva en los últimos años. La inclusión de la equidad de género es el resultado de un largo proceso social y político, producto fundamental del esfuerzo del movimiento de mujeres, en el cual su asimilación en el debate público por otros actores está condicionada por el contexto político global y por las concepciones y valores vigentes. La equidad de género en la educación y particularmente en la educación superior constituye un tema relevante por su impacto en el desarrollo económico y social. En el caso de América Latina, el carácter excluyente que han tenido sus universidades como herencia de más 300 años de colonialismo, manifiesta la tradicional discriminación de género. (Baute, Pérez, \& Espinoza, 2017).

\section{En palabras de Mayorga (2018):}

El enfoque de igualdad de género en la Educación Superior responde esencialmente a la necesidad de alinear los conocimientos con la realidad, aprendizajes y potencialidades para que el profesional garantice su intervención en el ámbito laboral la igualdad de género en su desempeño. La búsqueda de la igualdad de género es un elemento central de una visión de la sostenibilidad en la cual cada miembro de la sociedad respeta a los demás y desempeña un papel que le permite aprovechar su potencial al máximo.

Esta misma autora, en base a una de sus fuentes, aporta un reciente concepto sobra la igualdad de géneros, que en síntesis alude a "la igualdad de derechos, responsabilidades y oportunidades de mujeres y hombres, niñas y niños" misma igualdad que es vista tanto como "una cuestión de derechos humanos" así como también "una condición previa para, y el indicador del desarrollo sostenible centrado en las personas".

Para efectivizar la dimensión de igualdad de género no se logra únicamente con cambios de la estructura de la política y recursos económicos, se debe cambiar la forma de pensar y actuar de los docentes en su dinámica de dar clases en articular la igualdad de género en sus contenidos, cambio de mentalidad con la función de generar concientización en sus alumnos de manera que se empoderen en el tema de igualdad y con su intervención profesional generen cambios positivos en la sociedad. (Mayorga, 2018)

Paralelamente, de la obra de Salinas (2018) se extrae que:

La universidad como espacio de formación integral, tiene la misión de brindar a los futuros profesionistas, espacios y recursos que les permitan no sólo capacitarse en un área del conocimiento, sino crecer como seres humanos. [...] El género, la identidad de género y al rol o papel de género son elementos vinculados a la sexualidad. El género es una categoría social con introyectos psicológicos; la identidad de género como el sentir psicológico íntimo de ser hombre o mujer, y rol o papel de género como comportamientos que se adoptan por demandas sociales vinculadas al sexo (Álvarez, 2011). [...] El poder es un elemento vinculado al género, que permea las relaciones e interacciones con el otro, y produce ciertas prácticas sociales, como aquellas actividades, conductas y actitudes atribuidas a los géneros y desarrolladas por hombres y mujeres que reproducen lo que socialmente se espera de ellos de acuerdo al género con el cual son identificados. Al respecto, Silvei- 
ra (2001) indica que el concepto de género se refiere a una: atribución de responsabilidades y una serie de expectativas distintas que desde la sociedad se deposita en hombres y mujeres. [...] (p. 181).

Más adelante este autor refiere que está implícita la relación de poder como temática cuando se quiere exponer sobre el de las relaciones de género y violencia, así como también la de las hipótesis y convicciones que se forjan alrededor de una aparente esencia femenina y masculina, el amor y la manera en que interrelaciona con la pareja. En efecto agregan que, por ejemplo, en un trabajo de (Ramírez \& Núñez, 2010) realizado con el objeto de detectar indicadores de violencia en la pareja, se obtuvo que

[...] la educación diferenciada en hombres y mujeres es un factor de riesgo para la violencia de género, ya que a los hombres les enseñan a ser autoritarios y a las mujeres abnegadas. Los resultados de los procesos de socialización pueden revertirse a través de la educación, de tal manera que se instalen nuevos aprendizajes y se cuestionen los que ya existían. (p. 182).

Seguidamente, hacen también referencia la obra de (Fernández, 2014) quien se inclinó en favor del "[...] trabajo preventivo en los espacios educativos para fomentar la igualdad, la equidad y el respeto a la diversidad, así como para erradicar la violencia de género en las parejas jóvene". (p. 182).

Otra opinión que se comparte y que por ende se considera valioso referir de estos especialistas, es lo expresado respecto a una forma común de expresión de desigualdad en el ambiente universitario, aludiendo consecutivamente a la importancia de la formación en género cuando describen que:

[...] En el contexto universitario podemos ver la desigualdad expresada en diversas formas, una de ellas la encontramos en la elección de carrera que hacen las mujeres, quienes siguen optando por carreras del área de las ciencias sociales y las huma- nidades, dejando de lado las carreras del área de las ciencias exactas. [...] La formación en género en la educación superior es importante porque se dota a los alumnos de un marco interpretativo de la realidad vinculado con la necesidad de construir sociedades más justas y democráticas. (p. 183).

En este mismo orden de ideas explican Solís, Fernández, Solís, \& Terán (2018), quienes citando a (Lober, 2010) inician aportando su concepción en cuanto a la desigualdad de género, indicando este que:

La desigualdad de género toma formas diferentes, dependiendo de la estructura económica y de la organización social de una Sociedad en particular y de la cultura de cualquier grupo particular dentro de esa sociedad; aunque se habla de desigualdad de género, suelen ser las mujeres quienes resultan desfavorecidas con respecto a los hombres (p. 15).

Con base en las manifestaciones de la (Comisión Europea, 2016) estos mismos seguidamente explican que:

Las actitudes hacia la igualdad están evolucionando, la generación más de hoy no es inmune a los estereotipos y disparidades de género; persisten las desigualdades de género en la educación, en términos de preferencias de los sujetos de estudio, desempeño y patrones de participación [...]

Adicionalmente, estos mismos citan a (Rodríguez y Limas, 2017) para destacar que:

La presencia de las mujeres (hombres) en algunos programas educativos puede facilitarse o restringirse de modo que una consecuencia consista en obstaculizar la incorporación de ellas (ellos) a ciertas opciones laborales creando brechas y diferencias en los salarios de algunos y limitando los niveles de ingresos de las otras (p. 16).

\section{Tendencia Mundial de feminización de la educación superior}

Sobre este aspecto Fuentes (2016) brinda 
una destacable opinión tras su experiencia y análisis del proyecto denominado "Fortalecimiento de la Equidad de Género en la Educación Superior en Colombia [FEGES]", fundamentándose en la revisión de fuentes secundarias, y en la reflexión en torno a normas, políticas y proyectos ejecutados en favor de la justicia de género en la educación terciaria. La especialista expone que:

Según la Unesco [2012], la educación terciaria experimentó un vertiginoso aumento en sus tasas de matrícula. Así, entre 1970 y el 2009, los estudiantes matriculados pasaron de 32 a 165 millones, respectivamente; es decir, hubo un crecimiento del $516 \%$. En este periodo la tasa bruta de matrícula masculina (TBM) pasó del $11 \%$ al $26 \%$, lo que equivale a un aumento del $236 \%$, mientras que la TBM femenina pasó del $8 \%$ al 28 $\%$, lo que representa un incremento del 350 $\%$. De allí que el informe de este organismo internacional concluya que las principales beneficiarias de la "extraordinaria" expansión de la educación superior hayan sido las mujeres. Otro indicador global importante es la evolución del índice de paridad entre los sexos (IPS), el cual subió del 0,74 con predominio masculino en 1970 al 1,09 en el 2009, lo que representa una evolución "espectacular" hacia la paridad (Unesco, 2012: 77). Así, desde hace varias décadas, las mujeres se están educando más que los hombres y obtienen mejores resultados. Sin embargo, el mercado laboral y los espacios políticos y socioculturales no reconocen "la revolución educativa" de las mujeres, lo que se manifiesta en que ellas enfrentan más obstáculos para ocupar puestos mejor remunerados y de mayor prestigio e incidencia social. (p. 67-68).

Ahora bien, de acuerdo con lo argumentado por Costa \& Tombesi (2019) bien podría decirse que dicha tendencia probablemente pudiera ser constatable con los datos aportados por el Foro Económico Mundial [WEF, por sus siglas en inglés], ya que en base al Índice Global de Brecha de Género creado por este importante organismo hace poco más de diez años, es posible saber que las mujeres en la región latinoamericana, en términos generales, han representado un significativo avance en diversos ámbitos, incluso por encima de las otras regiones a nivel mundial. Actualmente, este indicador se ubica en 0.8, un dato excepcional, considerando que, mientras este valor se acerque más a 1 (uno) significa la paridad, y si se acercase a 0 (cero) quiere decir que se plantea la desigualdad. Sin embargo, refieren las afirmaciones de Mario Castillo, responsable de la División de Asuntos de Género de la Comisión Económica para América Latina y el Caribe (Cepal), órgano regional del Consejo Económico y Social de la ONU, quien dijo:

Cabe destacar que en la última década ha habido bastante mejoría, especialmente en la educación de las mujeres, pero hay muchas cosas que los datos no muestran. La desigualdad es diferente entre los países, pero también dentro de cada país. Y persiste.

A parte de todo lo antes referido, y como se sabe ocurre con casi cualquier otro aspecto de la vida o punto de vista, no todo está dicho, pues siempre existirá una posición distinta con argumentos igualmente válidos de considerar. Vendría siendo entonces casos como el de Robinson (2018), quien en su aporte inicia aclarando que uno de sus objetivos es el de analizar:

[...] argumentos que sostienen que, alrededor del mundo, siguen existiendo relaciones de poder de género en varias áreas concretas, como la educación y la violencia. Dichos argumentos sugieren que la desigualdad de género es visible tanto en la esfera privada como en la pública (sobre todo en sus aspectos económicos, políticos y sociales), y aducen pruebas empíricas para algunos de los ejemplos de desigualdad de género más apremiantes. (p. 2).

Esta autora, para seguir sosteniendo el argumento de contravención a enfoques con perspectivas

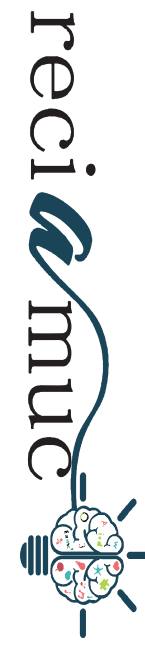


Lineales del progreso en materia de género, se apoya en fuentes tales como (UNWomen, 2015) organismo que asegura que:

[...] en general, son las mujeres las que llevan la peor parte de la pobreza, la desigualdad en el mercado de trabajo y la violencia, por ejemplo. De hecho, en el mundo las mujeres ganan, de media, el $24 \%$ menos que los hombres. (p. 3).

Así mismo, hace referencia al Informe de desigualdad mundial [WIR, por sus siglas en inglés] de 2018, ya que según esta, tratándose de "una evaluación sistémica de primer orden sobre la globalización en términos de desigualdad de renta y de riqueza" el mismo deja en evidencia que, a pesar del desarrollo sostenido experimentado en muchas economías emergentes, todavía existe un marcado aumento de la desigualdad económica mundial desde la década de los años 80, siendo en base a ello que la autora sentencia que "Es precisamente en este contexto donde hay que situar cualquier análisis de las desigualdades de género". (p. 3).

Respecto a Europa, esta vez con fundamento en la obra de (Boffey, 2017), continúa la entendida exponiendo que este, teniendo como referencia la última tabla comparativa sobre la desigualdad de género en la Unión Europea, se conoce que la igualdad de género en dicha región ha evolucionado paulatinamente entre 2005 y 2015.

\section{Contexto ecuatoriano sobre la igualdad en el sistema educativo superior}

En base a Mantilla, Galarza, \& Zamora (2017), apoyados a su vez en (Goetschel, 2007), podría decirse que, un hecho de la historia local que de manera particular marcó un hito para el género femenino en la educación superior del país, indiscutiblemente lo representa Matilde Hidalgo de Procel, al convertirse a principios del siglo XX en la primera médico del país.

Particularmente, es posible atreverse a ase- gurar que el Ecuador no es la excepción en cuanto a otra idea descrita por estos mismos tratadistas, en cuanto que es por la marcada cultura machista y tradiciones antiguas que preservan las diferencias intelectuales entre hombres y mujeres, lo que elementalmente genera, inclusive. el conflicto en las universidades, en la que adicionalmente se mezclan con diversos factores. De la misma manera, estos indicaron que:

Tanto en Ecuador como en otros países del mundo, el sexismo continúa siendo un problema social, cultural y político, lo que dificulta no solo las relaciones sociales de equidad entre los géneros, sino también la percepción de las capacidades asignadas a cada uno de ellos, otorgando en el ámbito académico la idea de que existen carreras masculinas y carreras femeninas. (Mantilla, Galarza, \& Zamora, 2017).

Hasta cierto punto escandaliza que, el escenario donde realmente avanzan materialmente la inclusión de disposiciones sobre igualdad de género fue el que propició el manifiesto de la Ley Orgánica de Educación Superior (LOES) en el año 2010, de su Reglamento General y en 2013 el del Reglamento de Régimen Académico debido a que reestablece y apertura un ambiente para la planificación, gestión y desarrollo del Sistema de Educación Superior. (Herdoíza, 2015).

Más adelante, esta misma asegura que, a pesar de lo antes expuesto, todavía perduran brechas de desigualdad social, profundizadas por múltiples variables, entre las que se encuentra la de género, que en síntesis perturban el ingreso, la constancia, y el egreso de las poblaciones discriminadas comúnmente, además de influir de manera negativa la práctica y la situación de la docencia, la gestión, los contenidos y prácticas académicas de las instituciones de educación superior.

\section{Hechos}

Para este aspecto se vuelve necesario co- 
menzar mencionando algunas de las iniciativas más destacadas que a nivel nacional se vienen implementando en materia de igualdad de género, desde un punto de vista general.

Es válido entonces referir que, si bien existe la articulación en el marco jurídico local, se considera más productivo indicar cuáles son algunas de las recientes ejecuciones que materialmente representan las instituciones del Estado ecuatoriano.

Con el apoyo de la Oficina de ONU Mujeres en Ecuador, el Ministerio de Relaciones Exteriores y Movilidad Humana de Ecuador (MREMH), recientemente ha generado el instrumento "Política para la Igualdad de Género" en el cual, inicialmente se deja en evidencia el compromiso del Gobierno Nacional de implementar la Agenda 2030 y los Objetivos de Desarrollo Sostenible (ODS), caracterizándose la primera de las mencionadas por la concepción de la igualdad de género como elemento central, mientras que dentro de los ODS, el quinto específicamente destaca por tratarse de la igualdad de género y al empoderamiento de las mujeres, niñas y adolescentes. El objetivo general del instrumento es el de:

Generar estrategias encaminadas a materializar la igualdad de género en todas las áreas de competencia del Ministerio de Relaciones Exteriores y Movilidad Humana, mediante la transversalización de la perspectiva de igualdad sustantiva en la gestión de las relaciones internacionales, la política exterior, la cooperación internacional, y la movilidad humana en todas las dependencias de esta institución a nivel nacional y en el exterior. (MREMH, 2018, pág. 14).

A nivel nacional, son varias las acciones encaminadas por diversas instituciones del Estado ecuatoriano en cuanto a la prosecución del equilibrio de los sexos a nivel de la educación superior, una muestra de ello bien podría ser lo auspiciado por el Consejo Nacional para la Igualdad de Género (CNIG) ante el Sistema se Educación Supe- rior (SES), velando porque la igualdad sea asumida como un importante criterio de medición de la eficacia de la educación.

Igualmente puede hacerse referencia a la creación de la Red de Educación Superior y Género en de abril de 2015, a la cual se acogieron 43 de las 59 universidades y escuelas politécnicas nacionales, mismas que bajo la articulación de importantes instituciones como la Secretaría Nacional de Planificación y Desarrollo (Senplades), el Consejo de Evaluación, Acreditación y Aseguramiento de la Calidad de la Educación Superior (Ceaaces); el Ministerio Coordinador de Conocimiento y Talento Humano (Mccth), la Secretaría de Educación Superior, Ciencia, Tecnología e Innovación (Senescyt); el Consejo Nacional para la Igualdad de Género (CNIG), la Agencia de las Naciones Unidas para la Igualdad de Género y el Empoderamiento de la Mujer ONU Mujeres (UN WOMEN - Ecuador) y la facultad latinoamericana de ciencias sociales (FLACSO); estipulan la responsabilidad integral que debe ser asumida por las mencionadas Casas de Estudios de garantizar la igualdad no solamente en sus propios dominios estudiantiles, docentes, directivos y administrativos, sino además en los contenidos de sus mallas curriculares y en la generación de conocimientos. (Senescyt, 2015).

\section{En la UG}

Ahora bien, ha sido precisamente a partir de ese mismo año 2015 que la UG ha avanzado particularmente en materia de equilibrio de género, y esto podría argumentarse, por una parte, cuando pone a disposición su Centro de Equidad de Género que, surgido de la Gestión del Vicerrectorado de Investigación, Postgrado y Gestión del Conocimiento, es creado a fines de que se encargue del facilitamiento de servicios de orientación y atención psicológica, asesoría legal y atención en casos de discriminación (indistintamente del sexo de la presunta víctima), aparte de encargarse de otros aspec- 
tos capacitación y asociación. (Universidad de Guayaquil, 2018).

Con fundamento en la revisión de la literatura efectuada para este trabajo, es posible inferir que parte fundamental de la gestión universitaria se centró en un plan de sensibilización denominado "Tejiendo caminos hacia la igualdad de género", proceso que tuvo como población objetivo todo el alumnado de la UG, pues así se ha evidenciado en varios trabajos de grado aportados por el propio alumnado en varias de sus facultades y, sobre todo, en investigaciones de hace apenas dos años.

Por mencionar algunos, a manera de ejemplo, está el aporte de López (2017), quien se propuso evaluar los logros y dificultades del Proceso de Sensibilización en Violencia de Género y Sexual en la facultad de ciencias económicas de la Universidad. Tras su análisis logra identificar "[...] que en los espacios universitarios existe violencia de género por parte de partidos políticos que existen en la Facultad de Ciencias Económicas [ ...]" aclarando que en dichos grupos se admite la participación femenina porque así lo establece el reglamento, sin embargo, "[...] no se les da mucha participación en el partido político [...]" y finalmente agregando que el machismo aún se hace evidente. (p. 55).

En síntesis, la autora alcanza definir como logro la sensibilización de "... ciento cincuenta y cuatro estudiantes de la Facultad de Ciencias Económicas..." a quienes se les hizo conocer "...las causas y consecuencias de la violencia de género [...] generaron un aprendizaje significativo que fue socializado con familiares, compañeros (as), amigos (a), debido a que reconocen la importancia de prevenir y sancionar cualquier tipo de violencia basada en género"; mientras que las dificultades identificadas se caracterizaron por el hecho de encontrarse con la "... resistencia por parte de los docentes para dar su hora de clases en actividades ajenas a la cátedra" (p. 63).
Otro trabajo es válido citar es el de Ramírez (2017), quien intervino a cuatro grupos de estudiantes con la aplicación un plan de sensibilización, mediante la facilitación de talleres a dichos grupos del ciclo I la Facultad de Ciencias Administrativas de la misma casa de estudios, logrando identificar no solo comportamiento como factores de vulnerabilidad ante la violencia de género en estudiantes universitarias, desde un enfoque de género, sino además deja en evidencia la participación que funge la sociedad en general respecto a "... las problemáticas sociales y las relaciones del ser y del quehacer de lo masculino y lo femenino dentro de contextos determinados, impulsando con ello comportamientos que atentan en un momento dado contra la salud física y mental de las estudiantes." (p. 1). Los factores de vulnerabilidad encontrados fueron;

a) factores de riesgos: antecedentes de violencia familiar, bajo nivel socioeconómico, baja autoestima, lucha de poderes, sumisión y dependencia; b) Situaciones de violencia de género que se repiten con frecuencia: relaciones de parejas conflictivas, violencia verbal entre docentes y estudiantes, discriminación a estudiantes embarazadas por docentes, preferencia hacia los hombres por parte de la administración por considerar que pueden cuidar mejor los equipos; c) al relacionar los factores se encontró: existe antecedentes de violencia marital y familiar, provienen de niveles socioeconómicos bajos, hay violencia ocasionada por los compañeros y docentes, lo que lleva a la mujer a ser sumisa, dependiente del hombre e incluso esto llevo a que desertaran de la carrera universitaria. (p. 61-62).

Para finalizar, puede indicarse que una de las últimas actuaciones indefectibles que consecuentemente ha protagonizado la gestión actual de la UG viene siendo la adopción y complementación del "Protocolo de prevención y actuación en casos de acoso, discriminación y violencia basada en género en la Universidad de Guayaquil" 
en el presente año 2019.

Este reciente instrumento procedimental, emanado en razón de antecedentes de desigualdad de género, discriminación y violencia de género, por una parte, y ante la disposición resolutoria del Consejo de Educación Superior (CES) al instruir su implementación, por la otra, inicialmente le fue encomendado preparar a la plantilla del Vicerrectorado de Bienestar Estudiantil (ViBe), vigente a junio de 2018, y esta dependencia , a su vez, encarga la revisión y adaptación de dicho ordenamiento (por cierto, originalmente formulado por Senescyt) a la Jefatura de Consejería y Orientación Académica del Vicerrectorado de Bienestar Estudiantil, para que fuera ejecutado según las condiciones estructurales y situacionales de la UG. (ViBe, 2019).

Resulta ser que, posteriormente, la Comisión de Intervención y Fortalecimiento Institucional (CIFI), considerando la multicausalidad y complejidad de la problemática, así como el amplio espectro que alcanza dicho protocolo en cuanto a los agentes interventores en la comunidad universitaria, resuelve la conformación de una comisión interdisciplinaria, a fines de asegurar un enfoque sistémico, serio y rápido en la identificación de los comportamientos ante situaciones de acoso, discriminación y violencia de género que transgreden los derechos a una educación y ambiente laboral libre de violencia. Indudablemente debe asumirse que este marco normativo:

[...] se sustenta en la normativa internacional, nacional e institucional vigente y de derechos humanos de las mujeres, así como en la revisión de varios instrumentos de prevención y erradicación de la violencia de género en el ámbito universitario nacional e internacional. (p. 3).

Su objetivo es:

[...] establecer acciones y procedimientos de actuación frente a situaciones de violencia, acoso, discriminación y violencia ba- sada en género, contra cualquier miembro de la Comunidad Universitaria, con el fin de garantizar la prevención, atención oportuna, protección, investigación y restitución inmediata de los derechos vulnerados, de manera rápida, ágil y eficaz y garantizando la integridad y la no revictimización de las víctimas, con las personas agresoras. (p. 4).

Los modos de actuación dispuestos obedecen a nueve principios rectores que son: i) Confidencialidad; ii) Profesionalismo; iii) Imparcialidad; iv) No re-victimización; v) Integralidad; vi) Transversalidad de género y enfoque de derechos; vii) Principio Pro ser humano; viii) Equidad e Inclusión y ix) Debida diligencia.

\section{Conclusiones}

Luego de todo el proceso de revisión y análisis de las distintas ideas referenciadas, el equipo ponderó completa e indiscutiblemente retomar el argumento de Mayorga (2018) para iniciar esta sección, debido a que la misma explica que:

La búsqueda de la igualdad de género es un elemento central de una visión de la sostenibilidad en la cual cada miembro de la sociedad respeta a los demás y desempeña un papel que le permite aprovechar su potencial al máximo. La amplia meta de la igualdad de género es una meta social a la que la educación y las demás instituciones sociales deben contribuir. La discriminación de género está imbricada en el tejido de las sociedades. La vinculación de la educación superior con la igualdad de género es un enfoque con la finalidad de crear revolución en los cambios de la mentalidad de los estudiantes y futuros profesionales con el afán de generar una sociedad justa, igualdad de derechos, las universidades. Los profesores son la clave para la fomentación en clases sobre la igualdad de género y los alumnos para la sociabilización de manera externa con su ejemplo y abasto conocimiento de derechos de igualdad de género. (Mayorga, 2018). 
Una alternativa bien podría ser ofertar dentro del pensum universitario la "Equidad de géneros" como asignatura electiva, aspecto en el que se desarrolló el estudio de Salinas (2018), quien respecto al objetivo de esta unidad de aprendizaje explicó que busca producir la reflexión entre todos los estudiantes de dos formas diferentes, primero, procurando alcanzar la desnaturalización de ciertas dinámicas de relación desiguales entre los géneros, así como la visibilización de diversas formas de violencia de género, y segundo, intentando lograr el entendimiento del propio papel, tanto en la construcción y mantenimiento de la desigualdad, como en el proceso de cambio, es decir, el proceso de construcción de equidad e igualdad.

Se vuelve igualmente trascendente reconocer dos aspectos indiscutibles de este problemática, pudiendo ser el primero de ellos, el hecho de que, efectivamente, tanto las políticas y estrategias gubernamentales como las de la UG, son percibidas como positivas y bien encaminadas hacia una mejoría en cuanto al cambio del enfoque del sistema educativo superior en favor del equilibrio de género, la inclusión y consideración de las mujeres en diversas esferas socioculturales, políticas y económicas, primordialmente. Sin embargo, el otro aspecto que también ha de reconocerse es que, aún y con la tendencia mundial, ni siquiera es soló Ecuador, sino toda Latinoamérica está muy lejos de materializar la abanderada transversalidad de género, puesto que la cuestión es, más que todo, de arraigo cultural en favor de una mentalidad machista o antifeminista que, por demás, es dura de desarticular en la mentalidad de la mayoría de los locales, que aun demuestra ser reacia a aceptar que la mujer es tan capaz y productiva que el hombre, muchas más veces de las que se reconoce.

\section{Bibliografía}

Baute, M., Pérez, V., \& Espinoza, M. (Diciembre de 2017). Estrategia de equidad de genero en el entorno universitario. Universidad y Sociedad, 9(4), 50-57.
Costa, C., \& Tombesi, C. (2019). Día de la Mujer: 6 gráficos que muestran cómo avanzaron (o no) las mujeres en América Latina. Inglaterra: BBC. Recuperado el 02 de 11 de 2019, de https://www.bbc. com/mundo/noticias-america-latina-47490978

Fuentes, L. (Abril de 2016). ¿Por qué se requieren políticas de equidad de género en la educación superior? Nómadas, 44(4), 65-83. Obtenido de http://nomadas.ucentral.edu.co/nomadas/pdf/nomadas_44/44_4F_La_equidad_de_genero_en_la_ educacion.pdf

Herdoíza, M. (2015). Construyendo igualdad en la educacion superior. Recuperado el 02 de 11 de 2019, de Universidad Estatal de Bolívar : http:// www.ueb.edu.ec/sitio/images/PDF/VICERRECTORADO-ACADEMICO/SISTEMA-EVALUACION-ESTUDIANTIL/CONSTRUYENDO-IGUALDAD-EN-LA-EDUCACION-SUPERIOR.pdf

López, D. (2017). Logros y dificultades del Proceso de Sensibilización en Violencia de Género y Sexual, facultad de ciencias económicas, Universidad de Guayaquil. Universidad de Guayaquil, Facultad de Ciencias Psicológicas. Guayaquil: Universidad de Guayaquil. Recuperado el 01 de 11 de 2019, de http://repositorio.ug.edu.ec/bitstream/redug/25265/1/L\%c3\%93PEZ_SALAZAR_ DEYSE_SUGEY_SISTEMATIZACI\%c3\%93N\%20 DE\%20EXPERIENCIAS.pdf

Mantilla, L., Galarza, J., \& Zamora, R. (2017). La inserción de la mujer en la educación superior ecuatoriana: caso Universidad Técnica de Ambato. Revista Latinoamericana de Estudios Educativos, 13(2), 1. Obtenido de https://www.redalyc.org/jatsRepo/1341/134154501002/html/index.html

Maqueira, G., \& Recalde, A. (Febrero de 2017). El modelo educativo de la Universidad de Guayaquil. Una experiencia inclusiva. Lecturas: Educación Física y Deportes, Revista Digital, 21(225), 1-14.

Mayorga, K. (2018). Igualdad de genero en la educación superior en el Siglo XXI. Palermo Business, 1(18), 137-144.

MREMH. (Febrero de 2018). Política para la igualdad de género. Recuperado el 02 de 11 de 2019, de Ministerio de Relaciones Exteriores y Movilidad Humana: https://www.cancilleria.gob.ec/wp-content/uploads/2018/02/politica_para_la_igualdad_ de_genero_2018.pdf

Ramírez, O. (2017). Factores de Vulnerabilidad ante la violencia de género en estudiantes del sexo. Universidad de Guayaquil, Facultad de Ciencias Administrativas . Guayaquil: Universidad de Guayaquil. Recuperado el 02 de 11 de 2019, de http:// repositorio.ug.edu.ec/bitstream/redug/25898/1/ 
Sistematizaci\%C3\%B3n\%20final\%200mar\%20 Ram\%C3\%ADrez\%20Romero.pdf

Robinson, V. (2018). Desigualdades de género«pasados" y futuras posibilidades. (BBVA, Ed.) Recuperado el 02 de 11 de 2019, de Open Mind BBVA: https://www.bbvaopenmind.com/wp-content/ uploads/2018/12/BBVA-OpenMind-Victoria-Robinson-Desigualdades-de-genero-problemas-pasados-y-futuras-posibilidades.pdf

Salinas, C. (Diciembre de 2018). Equidad de géneros como unidad de aprendizaje en la universidad. Alteridad, 13(2), 180-191.

Senescyt. (9 de Abril de 2015). Universidades sellan su compromiso para trabajar por la igualdad de género, Electrónica. Recuperado el 02 de 11 de 2019, de Secretaría de Educación Superior, Ciencia, Tecnología e Innovación: https://www. educacionsuperior.gob.ec/universidades-seIlan-su-compromiso-para-trabajar-por-la-igualdad-de-genero/\#
Solís, G., Fernández, M., Solís, L., \& Terán, C. (2018). Desigualdad de género en procesos educativos incidente en violencia hacia la mujer. Podium(33), 13-24.

Universidad de Guayaquil. (03 de Febrero de 2018). Centro de Equidad de Género. Recuperado el 02 de 11 de 2019, de Universidad de Guayaquil: http://www.ug.edu.ec/centro-de-equidad-de-genero/

ViBe. (30 de Julio de 2019). Protocolo de prevención y actución en casos de acoso, discriminación y violencia. (V. d. Guayaquil, Ed.) Recuperado el 02 de 11 de 2019, de Universidad de Guayaquil: http://www.ug.edu.ec/secretaria-general-r/ normativa/vigente/PROTOCOLO\%20DE\%2OPREVENCION\%20Y\%20ACTUACION\%20EN\%20 CASOS\%20DE\%20ACOSO\%2C\%20DISCRIMINACION\%20Y\%2OVIOLENCIA\%2OBASADA\%20 EN\%20GENERO\%20EN\%20LA\%20UNIVERSIDAD\%20DE\%20GUAYAQUIL\%202019.pdf

\section{CITAR ESTE ARTICULO:}

Loaiza Massuh, E., Salazar Torres, P., \& Romero Izurieta, R. (2020). El alumnado de la Universidad de Guayaquil (UG). Un vistazo al equilibrio de los sexos. RECIAMUC, 4(1), 229-241. doi:10.26820/reciamuc/4.(1).enero.2020.229-241

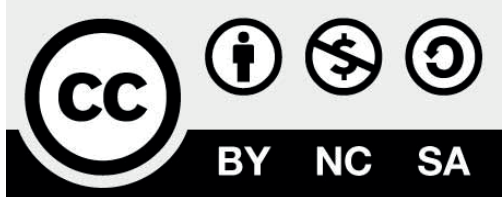

RECONOCIMIENTO-NOCOMERCIAL-COMPARTIRIGUAL CC BY-NC-SA

ESTA LICENCIA PERMITE A OTROS ENTREMEZCLAR, AJUSTARY CONSTRUIR A PARTIR DE SU OBRA CON FINES NO COMERCIALES, SIEMPRE Y CUANDO LE RECONOZCAN LA AUTORÍA Y SUS NUEVAS CREACIONES ESTÉN BAJO UNA LICENCIA CON LOS MISMOS TÉRMINOS. 